\title{
Precision Physics with the ATLAS detector at LHC
}

\author{
Pascal Pralavorio* ${ }^{*}$ \\ CPPM, CNRS/IN2P3 - Univ. Méditerranée, Marseille, France \\ E-mail: pralavor@cppm.in2p3.fr
}

\begin{abstract}
ATLAS is a general purpose detector that will start to take data at the CERN proton-proton collider LHC in 2007. Already in the initial luminosity phase $\left(10^{33} \mathrm{~cm}^{-2} \mathrm{~s}^{-1}\right)$, many physical quantities (masses, asymmetries, couplings) can be assessed with unprecedent statistical precision. The physical interpretation of the results will be driven by the understanding of the systematics coming from the detector performance and the event generation. Examples of expected ATLAS sensitivities in heavy gauge boson and top physics, mainly taken from the most recent simulation studies, are discussed.
\end{abstract}

International Europhysics Conference on High Energy Physics

July 21st - 27th 2005

Lisboa, Portugal

\footnotetext{
* Speaker.

On behalf of the ATLAS Cobllaboration
} 


\section{Introduction}

ATLAS (A Toroidal LHC Apparatus) [1] is a general purpose experiment located on the CERN proton-proton facility LHC (Large Hadron Collider), that will collide beams with a center of mass energy of $14 \mathrm{TeV}$ at a design luminosity of $10^{34} \mathrm{~cm}^{-2} \mathrm{~s}^{-1}$. During a first phase, the initial luminosity will be about 10 times lower, corresponding to $10 \mathrm{fb}^{-1}$ per year, and the jet activity reduced. This will be an ideal place to perform precision measurements thanks to the very high rate of Standard Model (SM) processes: 150, 15 and 11 millions of $W, Z$ and top events will be produced per year. The key issues for precise SM measurements are: very good lepton and jet energy scales to reduce systematics, high $b$-tagging performance to reduce QCD background and good precision on machine luminosity for cross-section measurements. From test beam results ${ }^{1}$, the former will be known to $1 \%$ and $10 \%$ respectively at the beginning and ultimately to $0.02 \%$ and $1 \%$ after in-situ calibrations with large samples of $Z \rightarrow l l$ and $Z+$ jets events. Recent full simulation studies show that a $b$-jet efficiency of $60 \%$ coupled with a light $(c)$ jet rejection above $100(10)$ is possible [3]. Finally, a precision around $2 \%$ on machine luminosity seems feasible by exploiting elastic $p p$ scattering in the Coulomb region [4].

\section{QCD and gauge boson physics}

The first task of ATLAS will be to understand events with QCD jets, as they represent the major source of background to most physic analyses. In this respect, the LHC prediction of minimum bias level is not reliable. The Monte-Carlo tuning will be done with a few thousands of LHC commissioning events and simple variables like the number of charged tracks. The main limitation comes from the track efficiency at low transverse momentum $\left(p_{T}<500 \mathrm{MeV}\right)$ [5]. A solution could be to take special ATLAS runs with no central magnetic field. As for minimum bias, the knowledge of proton parton distribution function (PDF), especially in the unprobed low $x$ region, will be essential for all SM processes. It can be accessed with the $W \rightarrow e v$ rapidity spectrum as, at LHC, the sea quarks are responsible of the $W$ production $\left(10^{-4}<x<0.1\right.$ and $\left.Q^{2}=M_{W}^{2}\right)$ [6].

One of the main motivation of gauge boson physics is a very precise measurement of the $W$ mass $\left(\mathrm{M}_{W}\right)$ to constraint the Higgs mass $\left(\mathrm{M}_{H}\right)$. The combined $\mathrm{M}_{W}$ measurements will be around $30 \mathrm{MeV}$ in 2007. The error on $\mathrm{M}_{W}$ needed to keep an equal contribution to $\mathrm{M}_{H}$ uncertainty, as a function of the error on top mass $\left(\mathrm{M}_{t}\right)$ is given by $\Delta \mathrm{M}_{W}=0.007 \times \Delta \mathrm{M}_{t}$ : so if $\Delta \mathrm{M}_{t}<2 \mathrm{GeV}$, this implies $\Delta \mathrm{M}_{W}<15 \mathrm{MeV}$, i.e. below $20 \mathrm{MeV}$ per LHC experiment. At hadron colliders, $\mathrm{M}_{W}$ is measured with the $W$ transverse mass in the channel $W \rightarrow l v, l=e, \mu$, where longitudinal neutrino momentum is not needed. With $10 \mathrm{fb}^{-1}$ of data, 60 millions of background free signal events are kept after a simple selection criteria. The method minimizes a $\chi^{2}$ comparing data and $Z^{0}$ tuned Monte-Carlo samples. Systematic uncertainties, largely dominant, are driven by the $15 \mathrm{MeV}$ error coming from lepton $E, p$ scale $^{2}$. The realization of such a stringent requirement will be very challenging and represents a good benchmark for ATLAS. Summing all the other contributions to $\Delta \mathrm{M}_{W}$ gives a total uncertainty lower than $20 \mathrm{MeV}$.

\footnotetext{
${ }^{1}$ A complete slice of ATLAS barrel with final electronics and DAQ was exposed to beams in 2004 [2].

${ }^{2}$ The central magnetic field known to $0.1 \%$, the inner detector aligned to $1 \mu \mathrm{m}$, the tracker material known to $1 \%$.
} 

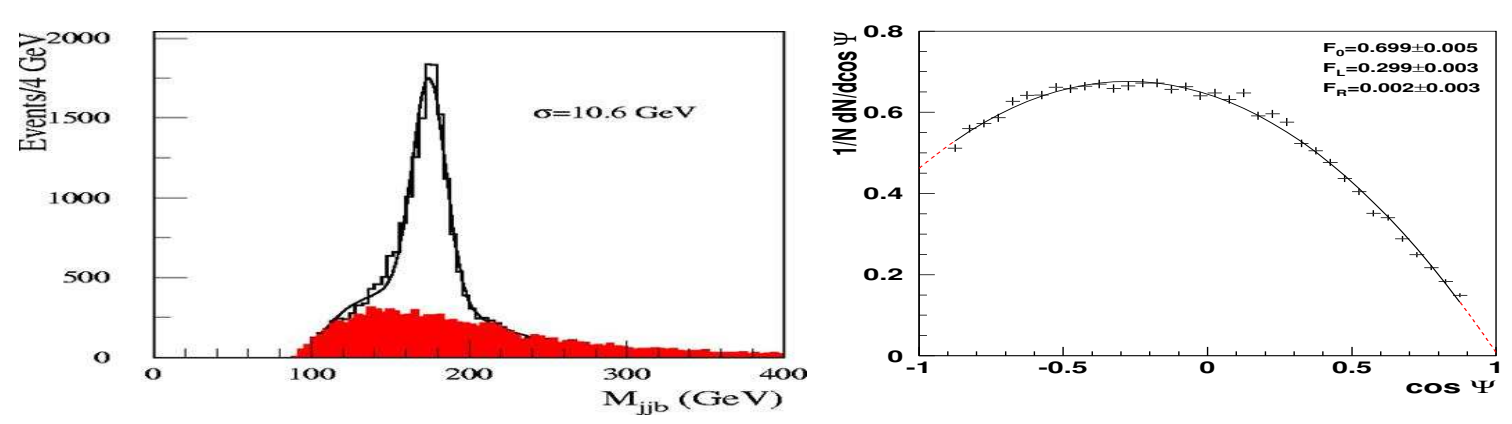

Figure 1: Left: Reconstructed top mass and combinatorial background (shaded area). Right: Reconstructed angular distribution for $W$ polarization with a fit superimposed. Both plots: semileptonic $t \bar{t}$ events, $10 \mathrm{fb}^{-1}$.

The study of Triple Gauge Couplings (TGC) provides a direct test of the non-abelian structure of the SM gauge group. There are split in SM allowed charged TGC ( $W W \gamma$ and $W W Z$ ) and SM forbidden neutral TGC ( $Z Z \gamma$ and $Z Z W)$. These TGC can be probed at LHC thanks to the high statistics, $>10^{6}$ di-boson events after $10 \mathrm{fb}^{-1}$, and the enhancement of anomalous couplings (ATGC) with center of mass energy. The most sensitive observable to ATGC at LHC is the high energy tail of $p_{T}^{Z}\left(p_{T}^{\gamma}\right)$ distribution. The sensitivity to ATGC, still dominated by the statistics after $30 \mathrm{fb}^{-1}$, improves compared to the current LEP limits by typically a factor 2-10 for the charged TGC parameters [7] and more than a factor 1000 for the neutral TGC [8].

\section{Top physics}

LHC will produce 8 (3) millions of $t \bar{t}$ pairs (single top) per year at initial luminosity. As all top quark decays through $t \rightarrow W^{+} b$, semileptonic (30\%), dileptonic (5\%) and all hadronic (65\%) $t \bar{t}$ final states are possible. Electroweak single top quark events are produced at $70 \%$ in the $t$-channel ( $W g$ fusion), and $25 / 5 \%$ in the $s$-channel $\left(W t / W^{*}\right)$. All these channels, except the all hadronic, are triggered by isolated lepton(s) and selected with 2,3 or 4 high $\mathrm{p}_{T}$ jets of which 1 or 2 are $b$-tagged, and missing energy. Selected semileptonic and dileptonic $t \bar{t}$ channels are background free, while single top channels (especially $W t$ and $W^{*}$ ) are dominated by $W+$ jets and $t \bar{t}$ background.

Precision measurements are thus performed in the $t \bar{t}$ channels. The full event topology is reconstructed after estimating the unknown neutrino(s) momentum(a) and correctly associate the two $b$-jets to the $b$ and $\bar{b}$ quarks. Various methods are exploited to perform top mass measurements [9]. The most promising is obtained in the semileptonic channel with the three jet invariant mass from the top (Figure 1 left). The measurement is dominated by the uncertainties on the $b$-jet energy scale and the final state radiation (FSR). They can be reduced with a kinematic fit on the full reconstructed event. The total error on the top mass is around $1 \mathrm{GeV}$.

Using the same fully reconstructed $t \bar{t}$ events, it is possible to test the top quark production and decay by measuring the SM predicted, but never measured, top spin correlation ${ }^{3}$, with asymmetries between spin-like and spin-unlike pairs [10]. By testing only the top decay, the $W$ boson polarization measurement complements these top spin studies. These two measurements are di-

\footnotetext{
${ }^{3}$ At LHC, $t$ and $\bar{t}$ spins are not polarized in $t \bar{t}$ pairs, but rather correlated.
} 


\begin{tabular}{|l||c|c|c||c|c|}
\hline Parameters & $F_{0}$ & $F_{L}$ & $F_{R}$ & $A$ & $A_{D}$ \\
\hline SM values & 0.703 & 0.297 & 0.000 & 0.422 & -0.290 \\
\hline Statistical errors & 0.004 & 0.003 & 0.003 & 0.014 & 0.008 \\
\hline Systematic errors & 0.015 & 0.024 & 0.012 & 0.023 & 0.010 \\
\hline
\end{tabular}

Table 1: ATLAS sensitivity to $W$ polarization components $\left(F_{0}, F_{L}, F_{R}\right)$ and top spin correlation asymmetries (A and $A_{D}$ ) after $10 \mathrm{fb}^{-1}$ and combination of dileptonic and semileptonic channels [11].

rectly accessible through angular distributions in top and $W$ rest frames, as illustrated in Figure 1 right, where the longitudinal $\left(F_{0}\right)$, left $\left(F_{L}\right)$ and right $\left(F_{R}\right) W$ helicity state probabilities are extracted from a 2 parameter fit. A precision of 1-2\% is obtained for $F_{0}$ and $F_{R}$ and $4 \%$ for the top spin correlation asymmetries after $10 \mathrm{fb}^{-1}$ (Table 1 ). These results are dominated by the same systematics as for the top mass measurement. A deviation from SM values would point to a top spin different from $1 / 2$, a decay $t \rightarrow H^{+} b$ and/or anomalous $g t \bar{t}, t W b$ couplings.

The measurement of the single top cross-section $(\sigma)$ at LHC will complement its possible discovery (no evidence up to now from the Tevatron). The expected precision on $\sigma$, including only statistical and theoritical errors, is around $8 \%$ in each channel after $30 \mathrm{fb}^{-1}$. The main systematics will come from the luminosity and the background, but still need to be evaluated. These crosssection measurements allows to access the CKM matrix element $V_{t b}\left(\sigma \alpha\left|V_{t b}\right|^{2}\right)$ and in the $W^{*}$ channel may be sensitive to the 2HDM model at high $\tan \beta(>45)$ and Higgs mass $(>200 \mathrm{GeV})$ region, complementing nicely the Higgs direct search.

\section{Conclusions}

LHC will be a $W, Z$ and top factory, already after one year at initial luminosity $\left(10 \mathrm{fb}^{-1}\right)$. With this statistics, ATLAS can precisely measure SM parameters, $W$ and top mass around $20 \mathrm{MeV}$ and 1 $\mathrm{GeV}$ respectively, and accurately test the top production and decay by measuring the top spin correlation to $4 \%$ and the $W$ polarization to $1-2 \%$. All these expected sensitivities, completely dominated by the systematics, are 2 to 10 better than Tevatron expectations for run II and can therefore open a window on new physics. Other precision measurements will need more statistics $\left(30 \mathrm{fb}^{-1}, 100\right.$ $\mathrm{fb}^{-1}$ ) like the single top cross-section, or the search for anomalous triple gauge couplings.

\section{References}

[1] The ATLAS Collaboration, CERN/LHCC/1999-14-15 (1999).

[2] R. Teuscher in Detectors and Data Handling session.

[3] S. Corréard et al., ATL-PHYS-2004-006.

[4] ATLAS Collaboration, CERN/LHCC/2004-010.

[5] A. Moraes et al., ATL-PHYS-PUB-2005-007 and ATL-PHYS-PUB-2005-015.

[6] A. Tricoli et al., ATL-PHYS-CONF-2005-008 [hep-ex/0509002].

[7] M. Dobbs and M. Lefebvre, ATL-PHYS-2002-022 and ATL-PHYS-2002-023.

[8] S. Hassani, ATL-PHYS-2003-022 and ATL-PHYS-2003-023.

[9] I. Borjanovic et al., Eur. Phys. Jour. C 39 S2 (2005), 63 [hep-ex/0403021].

[10] W. Bernreuther et al., Nucl. Phys. B 690 (2004) 81 [hep-ph/0403035].

[11] F. Hubaut et al., Eur. Phys. Jour. C 44 S2 (2005), 13 [hep-ex/0508061]. 